\title{
Realizing the dream of reproductive medicine
}

\author{
Jie Qiao is a reproductive physician and biologist who is President and Chief Physician of Peking University Third \\ Hospital. She is also Director of the National Clinical Research Center on Obstetrics \& Gynecology and a member \\ of the Chinese Academy of Engineering. Additionally, Professor Qiao is President of China Women Doctors \\ Association and Chair for the Reproductive Medical Society of Chinese Medical Doctor Association.
}

Jie Qiao

. opened a letter with a picture of a baby. It said "Hello, Dr. Qiao. I am glad to tell you that my baby was born on March 6 . He's a boy, six and a half pounds, very healthy..." As a reproductive physician, the greatest happiness is to hear the good news of another healthy baby.

I deem myself a fortunate person. In 1981, I was admitted to Bejing Medical University. After graduation, I pursued my post-graduate degree in Obstetrics and Gynecology Department of Peking University Third Hospital (PUTH). My tutor was Professor Meizhi Li, who has been devoting herself to the research of polycystic ovary syndrome (PCOS), a fresh topic at that time. PCOS is one of the most frequent endocrine diseases in reproductive age women, affecting about $6 \%$ to $10 \%$ of them, and is a major cause for anovulatory infertility. At the same time, as a resident doctor, I managed the patients under the leadership of Professor Lizhu Zhang. Therefore, I witnessed the first in vitro fertilization (IVF) birth in mainland China in 1988, and also participated in part of the clinical process as the chief physician for the first IVF baby's mother. After my doctorate studies, I studied in the University of Hong Kong as a visiting scholar and then came to Stanford University Medical Centre for a post-doctoral course from 1997 to 2002. I was later formally appointed as the Chief of Reproductive Medicine Center of PUTH. On my return, I immediately initiated the establishment of the center's reproductive medicine laboratory, and led all staff in daily literature studies, reminding all the time to catch up on the most cutting edge research in the globe.

Our center has received more than half a million ambulatory visits, and conducted 17,484 fresh IVF cycles and

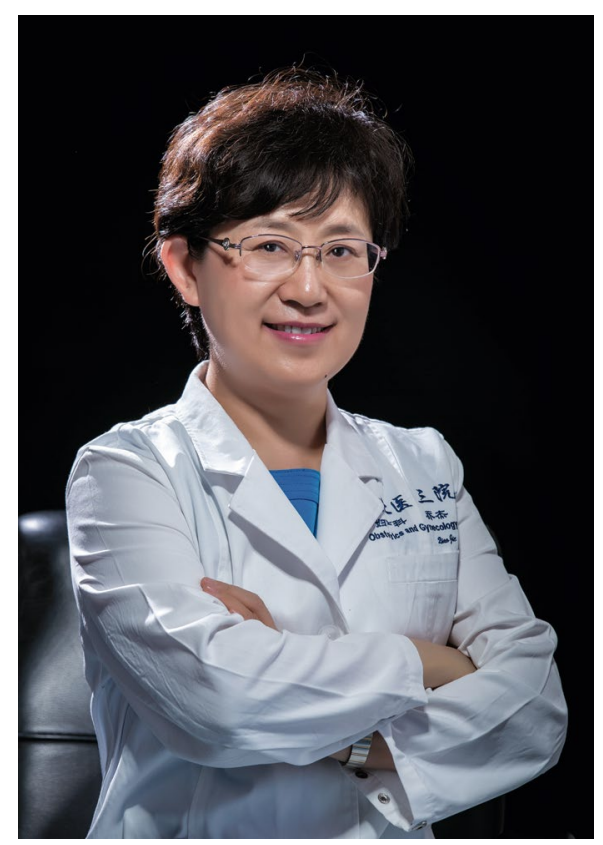

Credit: Jie Qiao

11,660 frozen embryo transfer cycles in 2018. Also, I led our clinical team in conducting investigations on the prevalence and the characteristics of PCOS in China, and proposing the features of hirsutism diagnostic criteria fitting the characteristics of the Chinese population. I have participated in the building of consensus on international hypertrichosis diagnosis and treatment, and it was adopted by the PCOS diagnostic standard in China. We uncovered the profiles of gut microbiota and its metabolites in the Chinese population of individuals with PCOS, and clarified their roles in the pathogenesis of PCOS. Our team also studied pregnancy outcomes in euthyroid women who have thyroid autoimmunity undergoing IVF with or without levothyroxine therapy, and found that the medication did not improve pregnancy rates in this group.

I focus on basic research and its clinical translation. Our team profiled the transcriptome and DNA methylome of human preimplantation embryos and germ cells, providing insights into the mechanism of epigenetic regulation during embryonic development. The insights from this research helped establish an innovative preimplantation genetic testing method, known as 'mutated allele revealed by sequencing with aneuploidy and linkage analyses' (MARSALA). Using next-generation sequencing with linkage analyses, it enabled simultaneous diagnosis of monogenic diseases and chromosome abnormality. So far, more than 200 families have been diagnosed using MARSALA and, after pre-pregnancy diagnoses, 80 healthy babies have been born.

I am grateful to the strong support from the government, and efforts of our staff and collaborators. We will continue our exploration in reproductive medicine.

\section{Jie Qiao $1,2,3,4,5$}

${ }^{1}$ Center for Reproductive Medicine, Department of Obstetrics and Gynecology, Peking University Third Hospital, Beijing, China. ${ }^{2}$ National Clinical Research Center for Obstetrics and Gynecology, Beijing, China. ${ }^{3}$ Key laboratory of Assisted Reproduction (Peking University), Ministry of Education, Beijing, China. ${ }^{4}$ Beijing Advanced Innovation Center for Genomics, Beijing, China. ${ }^{5}$ Peking-Tsinghua Center for Life Sciences, Peking University, Beijing, China. e-mail: jie.qiao@263.net

Published online: 9 September 2019 https://doi.org/10.1038/s41591-019-0558-4 